\title{
Weak Lefschetz theorems and the topology of zero loci of ample vector bundles
}

\author{
Shin-ICHi Matsumura
}

\begin{abstract}
The purpose of this paper is to study the fundamental group and higher homotopy groups of the zero locus of holomorphic sections of ample vector bundles on projective varieties, in the spirit of the Lefschetz hyperplane theorem. We give some Lefschetz type theorems by using Morse theory and the method of NapierRamachandran (the $L^{2}$-method and the theory of formal schemes).
\end{abstract}

\section{Introduction}

Positivity concepts in algebraic geometry and topological properties have been nourishing each other in the last decades. There are interesting results, such as the Lefschetz hyperplane theorem, the connectedness theorem of Fulton-Hansen, the Barth-Larsen theorem, and so on. In particular, the Lefschetz hyperplane theorem has been developed according to the context and the objectives, which says that the homotopy groups of a smooth projective variety $X$ can be compared with those of the zero locus of (holomorphic) sections of ample line bundles on $X$. This paper is devoted to the study of the topology of the zero locus of sections of ample vector bundles on smooth projective varieties, in the spirit of the Lefschetz hyperplane theorem. In this direction, Sommese gave the following celebrated result:

Theorem 1.1 (Proposition 1.16, [17], cf. Theorem 7.1.1, [10]). Let $E$ be an ample vector bundle of rank $r$ on a smooth projective variety $X$ of dimension $n$. Consider a section $s \in H^{0}\left(X, \mathcal{O}_{X}(E)\right)$ and the zero locus $S$ defined by

$$
S:=s^{-1}(0):=\left\{x \in X \mid s(x)=0 \in E_{x}\right\}
$$

Then we have

$$
H_{i}(X, S: \mathbb{Z})=0 \quad \text { for } i \leq n-r,
$$

where $H_{i}(X, S: \mathbb{Z})$ is a relative homology group. 
This theorem enables us to compare the homology groups $H_{i}(S: \mathbb{Z})$ and $H_{i}(X: \mathbb{Z})$ for $i \leq n-r$. If $E$ is an ample line bundle, we can obtain the same conclusion for the relative homotopy groups $\pi_{i}(X, S)$. Therefore, it is natural and of interest to ask whether or not the relative homotopy groups $\pi_{i}(X, S)$ vanish in the same situation as the above theorem. In this paper, we approach this problem under suitable positivity assumptions on vector bundles.

Problem 1.2 (The main problem). Under the same assumptions as in Theorem 1.1, does the relative homotopy group $\pi_{i}(X, S)$ vanish for $i \leq$ $n-r$ ?

In his paper [14], Okonek gave an affirmative answer for Problem 1.2, under the assumption that $E$ is an ample and globally generated vector bundle and $S$ has the expected codimension $r$. In his paper [9], Lazarsfeld proved that Problem 1.2 was affirmatively solved if $E \otimes B^{*}$ is globally generated for some ample and globally generated line bundle $B$, without assuming that $S$ has the expected codimension $r$. Here $B^{*}$ is the dual line bundle of $B$.

The following theorem asserts that Problem 1.2 is affirmatively solved under a weaker assumption than the above, which is one of the main results of this paper.

Theorem 1.3. Let $E$ be a vector bundle of rank $r$ on a smooth projective variety $X$ of dimension $n$. Consider a section $s \in H^{0}\left(X, \mathcal{O}_{X}(E)\right)$ and the zero locus $S$ of $s$. If the line bundle $\mathcal{O}_{\mathbb{P}\left(E^{*}\right)}(-1)$ is $(r+k-1)$-positive on $\mathbb{P}\left(E^{*}\right)$, then we have

$$
\pi_{i}(X, S)=0 \quad \text { for } i \leq n-r-k .
$$

In particular, the map (induced by the inclusion map $j: S \hookrightarrow X$ )

$$
j_{*}: \pi_{i}(S) \longrightarrow \pi_{i}(X)
$$

is isomorphic for $i<n-r-k$ and surjective for $i=n-r-k$.

Throughout this paper, $\pi: \mathbb{P}\left(E^{*}\right) \rightarrow X$ denotes the projective space bundle associated to the dual vector bundle $E^{*}$ of $E$ in the sense of 
Grothendieck, which is defined by

$$
\mathbb{P}\left(E^{*}\right):=\operatorname{Proj}\left(\bigoplus_{k=0}^{\infty} S^{k} E^{*}\right) \rightarrow X .
$$

Further $\mathcal{O}_{\mathbb{P}\left(E^{*}\right)}(-1)$ denotes the tautological line bundle on $\mathbb{P}\left(E^{*}\right)$, which has the natural inclusion $\mathcal{O}_{\mathbb{P}\left(E^{*}\right)}(-1) \rightarrow \pi^{*} E$. Namely, $\mathbb{P}\left(E^{*}\right) \rightarrow X$ is the fiber bundle with the space of the lines of $E_{x}$ as the fiber over $x \in X$ and $\mathcal{O}_{\mathbb{P}\left(E^{*}\right)}(-1)$ is the dual bundle of the hyperplane bundle on the fiber (which is isomorphic to $\mathbb{P}^{r-1}$ ). See Definition 2.3 for the definition of $q$-positive (q-ample) line bundles.

If $E$ is ample and globally generated, then $\mathcal{O}_{\mathbb{P}\left(E^{*}\right)}(-1)$ is $(r-1)$-positive (see [16, Proposition 1.3] and [6, Proposition 2.8]). We do not assume that $S$ has the expected codimension $r$ in the above theorem, thus Theorem 1.3 can be seen as a slight generalization of the results of $[9,14]$. For the proof, we apply Morse theory after constructing non-degenerate functions with suitable index by using Hermitian metrics obtained from the assumption on $\mathcal{O}_{\mathbb{P}\left(E^{*}\right)}(-1)$.

Problem 1.2 is closely related to a conjecture of Griffiths for ample vector bundles, which predicts that any ample vector bundle is Griffiths positive (i.e., the vector bundle admits a Hermitian metric with Griffith positive curvature). This conjecture was affirmatively solved in the special case of smooth curves (see [4]), but we do not know whether the conjecture is affirmative even in the case of surfaces. From simple computations, we can easily see that $\mathcal{O}_{\mathbb{P}\left(E^{*}\right)}(-1)$ is $(r-1)$-positive if $E$ is Griffiths positive (e.g., see [6, Proposition 2.8]). Therefore by Theorem 1.3 we know that Problem 1.2 is also affirmative only under the assumption that $E$ is ample, if the conjecture is affirmative.

Problem 1.2 is also related to the converse implication of the AndreottiGrauert vanishing theorem. The Andreotti-Grauert theorem asserts that any $q$-positive line bundle is always $q$-ample (see [1, Théorèm 14] and [6, Proposition 2.1]). In their paper [6], Demailly, Peternell and Schneider asked whether or not the converse implication of the Andreotti-Grauert theorem holds. It is a natural question, however it has been an open problem for a long time except the case of $q=0$ (see [11, 12] for more details).

By standard arguments on the spectral sequences of direct image sheaves, we can easily see that $E$ is ample if and only if $\mathcal{O}_{\mathbb{P}\left(E^{*}\right)}(-1)$ is $(r-1)$-ample on $\mathbb{P}\left(E^{*}\right)$ (see, e.g. [6, Example 2.7]). On the other hand, if $E$ is Griffiths positive, then $\mathcal{O}_{\mathbb{P}\left(E^{*}\right)}(-1)$ is $(r-1)$-positive. 
Theorem 1.3 implies that if the converse of the Andreotti-Grauert theorem for $\mathcal{O}_{\mathbb{P}\left(E^{*}\right)}(-1)$ holds, then Problem 1.2 is affirmative. Recently Ottem constructed a counterexample to the converse of the Andreotti-Grauert theorem, by investigating the Lefschetz hyperplane type theorem (see [15] for the precise argument), which gives a motivation to study Problem 1.2.

It seems to be difficult to handle higher homotopy groups. Here we give a partial result for the fundamental groups in Problem 1.2 (cf. [18]), which is one of the main results and a corollary of Theorem 3.6.

Corollary 1.4. Let $E$ be an ample vector bundle of rank $r$ on a smooth projective variety $X$ of dimension $n$ and $R$ be an analytic set of $X$. Consider a section $s \in H^{0}\left(X, \mathcal{O}_{X}(E)\right)$ and the zero locus $S$ of $s$. If $S$ has the expected codimension $r$ (i.e., $\operatorname{codim}_{X} S=r$ ) and $r<n$, then the map

$$
j_{*}: \pi_{1}(S \backslash R) \longrightarrow \pi_{1}(X \backslash R)
$$

is surjective.

In particular, if $R=\phi$, the map $j_{*}: \pi_{1}(S) \longrightarrow \pi_{1}(X)$ is surjective.

The proof is based on the method of Napier-Ramachandran and its variation. In their paper [13], Napier and Ramachandran constructed holomorphic sections of ample line bundles that separate sheets of an étale covering. We also construct such sections by using solutions of the $\bar{\partial}$-equation with bounded $L^{2}$-norm, which give an estimate of the index of the image of $\pi_{1}(S \backslash R)$ under $j_{*}$. Further, we compute this estimate by investigating the cohomology groups of formal completions. In this step, we apply the theory of formal schemes.

This paper is organized in the following way: In Section 2, we give a proof of Theorem 1.3. In Section 3, we give proofs of Theorem 3.6 and Corollary 1.4 .

\section{Lefschetz type theorems and Morse theory}

\subsection{Preliminaries}

In the proof of Theorem 1.3, we shall use Morse theory. For this reason, we collect materials on standard Morse theory in this subsection.

Let $\Phi$ be a real-valued smooth function on a compact $C^{\infty}$-manifold $M$ of (real) dimension $k$. A point $m \in M$ is called a critical point if $d \Phi=0$ at $m$. In terms of local coordinates $\left(x_{1}, \ldots, x_{k}\right)$, the (real) Hessian of $\Phi$ at 
$m \in M$ is defined by

$$
\left(\frac{\partial^{2} \Phi}{\partial x_{i} \partial x_{j}}(m)\right)_{1 \leq i, j \leq k}
$$

which is a symmetric quadratic form. If $m$ is a critical point of $\Phi$, the Hessian at $m$ does not depend on the choice of local coordinates. The number of negative eigenvalues of the Hessian at critical points is called the index. A function on $M$ is called non-degenerate if the function has only non-degenerate manifold. See [2, Definition 3.1] for the definition of nondegenerate manifolds. The following theorem asserts that non-degenerate functions allow us to reconstruct the manifold as $\mathrm{CW}$ complexes.

Theorem 2.1 (Theorem 3, [3]). Let $\Phi$ be a real-valued smooth function on a compact $C^{\infty}$-manifold $M$. Let $\lambda$ be the infimum of the indices of $\Phi$ at the critical points. Assume that $\Phi$ is non-degenerate. Then for an arbitrary real number $a, M$ is obtained from $W_{a}$ by successively attaching cells of dimension $\geq \lambda$, where $W_{a}$ is the subset defined by

$$
W_{a}=\{m \in M \mid \Phi(m)<a\}
$$

In particular, we have

$$
\pi_{i}\left(X, W_{a}\right)=0 \quad \text { for } i \leq \lambda-1
$$

For the proof of Theorem 1.3, we need suitable perturbations to construct non-degenerate functions. For this purpose, we apply the following approximation theorem. We take a finite open covering $\left\{U_{j}\right\}_{j=1}^{N}$ of $M$ by open balls $U_{j}$ with a coordinate $\left(x_{1}^{j}, \ldots, x_{k}^{j}\right)$. Further, we take a relatively compact set $V_{j}$ in $U_{j}$ such that $\left\{V_{j}\right\}_{j=1}^{N}$ is also an open covering of $M$. Then the $C^{2}$-norm $|\cdot|_{C^{2}}$ with respect to the open covering is defined to be

$\|f\|_{C^{2}}:=\sum_{j=1}^{N} \sum_{\alpha, \beta=1}^{k, k} \sup _{p \in \overline{V_{j}}}\left|\frac{\partial^{2} f}{\partial x_{\alpha}^{j} \partial x_{\beta}^{j}}(p)\right|+\sum_{j=1}^{N} \sum_{\alpha=1}^{k} \sup _{p \in \overline{V_{j}}}\left|\frac{\partial f}{\partial x_{\alpha}^{j}}(p)\right|+\sup _{p \in X}|f(p)|$

for every function $f \in C^{2}(M, \mathbb{R})$. A $C^{2}$-function is called $(\varepsilon, 2)$-small if the $C^{2}$-norm of the function is smaller than $\varepsilon$.

Theorem 2.2 ([18]). Let $\Phi$ be a real-valued smooth function on a compact $C^{\infty}$-manifold $M$. Then for an arbitrary positive number $\varepsilon>0$, there exists an $(\varepsilon, 2)$-small function $\eta_{\varepsilon}$ such that $\Phi+\eta_{\varepsilon}$ is non-degenerate on $M$. 


\subsection{Proof of Theorem 1.3}

This subsection is devoted to the proof of Theorem 1.3. For the proof, we shall construct non-degenerate functions with suitable index, from Hermitian metrics whose Chern curvature satisfies a partial positivity condition. By applying Morse theory to this function, we obtain the conclusion. First, we recall the definition of $q$-positive ( $q$-ample) line bundles.

Definition 2.3. Let $Z$ be a compact complex manifold of dimension $N$ and $q$ be an integer with $0 \leq q \leq(N-1)$.

(1) A line bundle $L$ on $Z$ is called q-positive, if there exists a (smooth) Hermitian metric $h$ whose Chern curvature $\sqrt{-1} \Theta_{h}(L)$ has at least $(N-q)$ positive eigenvalues at any point on $Z$.

(2) A line bundle $L$ on $Z$ is called $q$-ample, if for any coherent sheaf $\mathcal{F}$ on $Z$ there exists a positive integer $m_{0}=m_{0}(\mathcal{F})>0$ such that

$$
H^{i}\left(Z, \mathcal{F} \otimes \mathcal{O}_{Z}\left(L^{m}\right)\right)=0 \quad \text { for } i>q, m \geq m_{0}
$$

Proof of Theorem 1.3. Since $\mathcal{O}_{\mathbb{P}\left(E^{*}\right)}(-1)$ is an $(r+k-1)$-positive line bundle on $\mathbb{P}\left(E^{*}\right)$, there exists a smooth Hermitian metric $h$ such that the Chern curvature associated to $h$ has at least $(n-k)$ positive eigenvalues at any point on $\mathbb{P}\left(E^{*}\right)$. The metric $h$ can be seen as the function $F_{h}$ on the total space of $\mathcal{O}_{\mathbb{P}\left(E^{*}\right)}(-1)$ defined by

$$
F_{h}: \mathcal{O}_{\mathbb{P}\left(E^{*}\right)}(-1) \longrightarrow \mathbb{R}, \quad t \longmapsto|t|_{h}^{2},
$$

where $|t|_{h}$ is the norm of $t \in \mathcal{O}_{\mathbb{P}\left(E^{*}\right)}(-1)$ with respect to $h$. Since the total space of $\mathcal{O}_{\mathbb{P}\left(E^{*}\right)}(-1)$ is isomorphic to the blow-up of the total space of $E$ along the image of the zero section, we have the isomorphism

$$
\pi: \mathcal{O}_{\mathbb{P}\left(E^{*}\right)}(-1) \backslash \mathbb{P}\left(E^{*}\right) \stackrel{\cong}{\longrightarrow} E \backslash X
$$

By this isomorphism, the functions on $\mathcal{O}_{\mathbb{P}\left(E^{*}\right)}(-1) \backslash \mathbb{P}\left(E^{*}\right)$ can be identified with the functions on $E \backslash X$. In particular, $F_{h}$ can be regarded as the function on $E \backslash X$. A given section $s$ of $E$ can be seen as the holomorphic map from $X$ to the total space of $E$. We define the function $\Phi_{h}$ on $X$ by the pull-back 
of $F_{h}$ under the holomorphic map $s: X \backslash S \rightarrow E \backslash X$. Then $\Phi_{h}$ can be written as

$$
\Phi_{h}: X \backslash S \rightarrow \mathbb{R}, \quad x \longmapsto\left|\pi^{-1}(s(x))\right|_{h}^{2}
$$

The function $\Phi_{h}$ satisfies the following properties:

Claim 2.4. The function $\Phi_{h}$ defined above satisfies the following properties:

(1) $\Phi_{h}$ is a positive-valued smooth function on $X \backslash S$.

(2) The Levi-form $d d^{c} \Phi_{h}$ has at least $(n-r-k+1)$ negative eigenvalues at the critical points of $\Phi_{h}$.

Proof. It is sufficient to check the second property since the first property is obvious by the definition of $\Phi_{h}$. In this proof, we denote the line through a point $s(x) \in E_{x}$ by $[s(x)] \in \mathbb{P}\left(E^{*}\right)$. Since $\mathbb{P}\left(E^{*}\right) \rightarrow X$ is the fiber bundle with the space of the lines in $E_{x}$ as the fiber over $x \in X$, we have the natural projection $E \backslash X \rightarrow \mathbb{P}\left(E^{*}\right)$.

Fix an arbitrary point $p \in X \backslash S$. Let $W$ be a small open neighborhood of $p$ and $\bar{W}$ be the image of $s(W)$ under the projection $E \backslash X \rightarrow \mathbb{P}\left(E^{*}\right)$. Now we take a local frame $t$ of $\left.\mathcal{O}_{\mathbb{P}\left(E^{*}\right)}(-1)\right|_{\bar{W}}$ adapted at $[s(p)]$ with respect to the restriction $\left.h\right|_{\bar{W}}$ of $h$. For simplicity, we denote the restriction $\left.h\right|_{\bar{W}}$ by the same notation $h$. Namely, $t$ is a non-vanishing holomorphic map from $\bar{W}$ to $\left.\mathcal{O}_{\mathbb{P}\left(E^{*}\right)}(-1)\right|_{\bar{W}}$ such that

$$
|t([s(x)])|_{h}^{2}=1 \quad \text { and } \quad d\left(|t([s(x)])|_{h}^{2}\right)=0 \quad \text { at }[s(p)] .
$$

Since $W$ is isomorphic to $\bar{W}$, the composition $\pi^{-1} \circ s$ can be seen as the non-vanishing section of $\mathcal{O}_{\mathbb{P}\left(E^{*}\right)}(-1)$ on $\bar{W}$. Since $t$ is also a non-vanishing section on $\bar{W}$, there exists a non-vanishing holomorphic function $g$ on $W$ such that $\pi^{-1}(s(x))=g([s(x)]) t([s(x)])$ on $W$. Then by the definition of $\Phi_{h}$, we have

$$
\Phi_{h}(x)=|g([s(x)])|^{2}|t([s(x)])|_{h}^{2} \quad \text { on } W .
$$

From now on, we compute the Levi-form of $\Phi_{h}$. By simple computations, we obtain

$$
\begin{aligned}
\sqrt{-1} \partial \bar{\partial} \Phi_{h}= & \sqrt{-1}|t|_{h}^{2} \partial g \wedge \overline{\partial g}-\sqrt{-1} g \overline{\partial g} \wedge \partial|t|_{h}^{2} \\
& +\sqrt{-1} \bar{g} \partial g \wedge \bar{\partial}|t|_{h}^{2}+|g|^{2} \sqrt{-1} \partial \bar{\partial}|t|_{h}^{2}
\end{aligned}
$$


The second and third terms are equal to zero at $[s(p)]$ since $t$ is adapted at $[s(p)]$. Further it is easy to see that the first term is also equal to zero at $[s(p)]$ if $p$ is a critical point of $\Phi_{h}$. Indeed, we have

$$
\begin{aligned}
d \Phi_{h}(x) & =|t|_{h}^{2} d|g|^{2}+|g|^{2} d|t|_{h}^{2} \\
& =|t|_{h}^{2} d|g|^{2} \quad \text { at }[s(p)] .
\end{aligned}
$$

The second equality follows since $t$ is adapted at $[s(p)]$. Therefore if $p$ is a critical point of $\Phi_{h}$, then $d g=0$ at $[s(p)]$. From the above argument, we obtain

$$
\sqrt{-1} \partial \bar{\partial} \Phi_{h}(x)=|g|^{2} \sqrt{-1} \partial \bar{\partial}|t|_{h}^{2}
$$

if $p$ is a critical point of $\Phi_{h}$.

For the proof of the claim, we need to compute the number of negative eigenvalues of the Levi-form $\sqrt{-1} \partial \bar{\partial}|t|_{h}^{2}$. Since $t$ is a frame adapted at $[s(p)]$, we have

$$
\sqrt{-1} \partial \bar{\partial}|t([s(x)])|_{h}^{2}=\sqrt{-1} \partial \bar{\partial} \log |t([s(x)])|_{h}^{2} \quad \text { at }[s(p)] .
$$

By the definition of the Chern curvature, we have

$$
\sqrt{-1} \Theta_{h}=-d d^{c} \log |\tilde{t}|_{h}
$$

for any local frame $\tilde{t}$ of $\mathcal{O}_{\mathbb{P}\left(E^{*}\right)}(-1)$. Therefore $-\sqrt{-1} \partial \bar{\partial} \log |\tilde{t}|_{h}^{2}$ has at least $(n-k)$ positive eigenvalues. Since $\bar{W}$ is a (local) submanifold of codimension $(r-1)$ on $\mathbb{P}\left(E^{*}\right)$, the restriction of $-\sqrt{-1} \partial \bar{\partial} \log |\tilde{t}|_{h}^{2}$ to $\bar{W}$ has at least $(n-$ $r-k+1)$ positive eigenvalues, which implies that $\sqrt{-1} \partial \bar{\partial}|t([s(x)])|_{h}^{2}$ has at least $(n-r-k+1)$ negative eigenvalues at $[s(p)]$. This completes the proof of the claim.

We shall construct non-degenerate functions with suitable index by using Claim 2.4. First we choose sufficiently small neighborhoods $U, V \subset X$ of $S$ such that $V$ is relatively compact in $U$. Further, we take a smooth function $\rho_{V}$ such that $\rho_{V}$ is identically zero on $V$ and identically one on $X \backslash U$. Then the function $\rho_{V} \Phi_{h}$ is a smooth function on $X$. By applying the approximation theorem (Theorem 2.2) to $\rho_{V} \Phi_{h}$, for an arbitrary positive number $\varepsilon$, we obtain an $(\varepsilon, 2)$-small function $\eta_{\varepsilon}$ such that $\rho_{V} \Phi_{h}+\eta_{\varepsilon}$ is non-degenerate on $X$. Then we prove the following claim.

Claim 2.5. For a sufficiently small $\varepsilon>0$, the Levi-form of $\rho_{V} \Phi_{h}+\eta_{\varepsilon}$ has at least $(n-r-k+1)$ negative eigenvalues at the critical points of $\rho_{V} \Phi_{h}+$ $\eta_{\varepsilon}$ on $X \backslash U$. 
Proof. Since $\rho_{V}$ is identically one on $X \backslash U$, we have $\rho_{V} \Phi_{h}=\Phi_{h}$ on $X \backslash U$. Hence, the Levi-form of $\rho_{V} \Phi_{h}+\eta_{\varepsilon}$ has at least $(n-r-k+1)$ negative eigenvalues at the critical points of $\Phi_{h}$ by Claim 2.4, if $\varepsilon$ is sufficiently small. Since this is an open condition, there exists an open neighborhood $W$ of the critical points of $\Phi_{h}$ such that the Levi-form of $\rho_{V} \Phi_{h}+\eta_{\varepsilon}$ still has at least $(n-r-k+1)$ negative eigenvalues on $W$. It is easy to see that the critical points of $\rho_{V} \Phi_{h}+\eta_{\varepsilon}$ are contained in $W$ for a sufficiently small $\varepsilon$. Indeed, if $p$ is not a critical point of $\Phi_{h}$, then $p$ is not a critical point of $\rho_{V} \Phi_{h}+\eta_{\varepsilon}$.

We take a positive number $\delta$ with

$$
0<\delta<\inf \left\{\rho_{V}(x) \Phi_{h}(x)+\eta_{\varepsilon}(x) \mid x \in X \backslash V\right\}
$$

Then $W_{\delta}$ defined by

$$
W_{\delta}:=\left\{x \in X \mid \rho_{V}(x) \Phi_{h}(x)+\eta_{\varepsilon}(x)<\delta\right\}
$$

is contained in $U$. Since $\rho_{V} \Phi_{h}+\eta_{\varepsilon}$ is non-degenerate, we can apply standard Morse theory (Theorem 2.1). Then $X$ is obtained from $W_{\delta}$ by successively attaching cells of dimension $\geq n-r-k+1$. In particular, the natural map $\pi_{i}\left(W_{\delta}, S\right) \rightarrow \pi_{i}(X, S)$ is isomorphic for $i \leq n-r-k$. On the other hand, since $W_{\delta}$ is contained in $U$, we have the following commutative diagram:

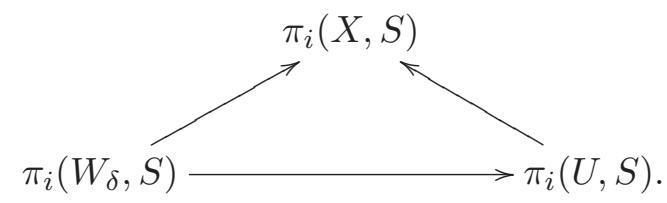

The diagonal map on the left is an isomorphism for $i \leq n-r-k$. We can choose $U$ to retract onto $S$ since $X$ can be triangulated with $S$ as subcomplexes. Then we have $\pi_{i}(U, S)=0$ for any $i$. Therefore, we obtain $\pi_{i}(X, S)=0$ for $i \leq n-r-k$. This completes the proof of Theorem 1.3.

\section{Weak Lefschetz type theorems}

\subsection{Key estimates}

In Section 3, we give proofs of Theorem 3.6 and Corollary 1.4. In the proof of Theorem 3.6, we apply the techniques established by Napier and Ramachandran in [13]. They proved that the image of the fundamental group of submanifolds with ample normal bundle is of finite index. The strategy 
of the proof can be divided into two steps. In the first step, they consider a suitable covering space and construct sections of ample line bundles that separate sheets of the covering. In the second step, they study the finite dimensionality of the space of the sections on formal schemes, which gives a bound on the index.

In this subsection, we give a proof of the following proposition (Proposition 3.1), which can be seen as a slight generalization of [13, Theorem 2.2]. The proof is based on a standard application of the $L^{2}$-method and is parallel to the proof of [13, Theorem 2.2]. For the reader's convenience, we give details of the proof.

Throughout this subsection, let $X$ be a projective variety of dimension $n, U$ be an open set of $X$ that does not intersect with the singular locus $X_{\text {sing }}$ of $X$ and $R$ be an analytic subset of $X$ containing $X_{\text {sing }}$. We consider an étale covering

$$
\pi: \widetilde{M} \longrightarrow M \text { and the degree } d:=\operatorname{deg} \pi,
$$

where $M$ is the complement $X \backslash R$. The following proposition gives an estimate of the degree $d$ by the dimensions of the spaces of the holomorphic sections. Let $L$ be a big line bundle and $G$ be an arbitrary line bundle on $X$. We set $E_{\nu}:=L^{\nu} \otimes G$ for a positive integer $\nu$, and we consider the space of the holomorphic $\pi^{*} E_{\nu}$-valued $(n, 0)$-forms

$$
H_{L^{2}(U \cap R)}^{0}\left(\widetilde{M}, \mathcal{O}_{\widetilde{M}}\left(\pi^{*} E_{\nu} \otimes K_{\widetilde{M}}\right)\right)
$$

with the following $L^{2}$-condition on $U \cap R$.

(*) For any point $r \in U \cap R$, there exists a neighborhood $V_{r}$ of $r$ and trivializations of $L$ and $G$ on $V_{r}$ such that

$$
\int_{\bar{V}_{r}} \sqrt{-1}^{n^{2}} s \wedge \bar{s}<\infty
$$

for any connected component $\overline{V_{r}}$ of $\pi^{-1}\left(V_{r}\right)$. We regarded $s$ as the holomorphic $(n, 0)$-form on $\pi^{-1}\left(V_{r}\right)$ under the fixed trivialization of $\pi^{*} E_{\nu}$. Here $K_{\widetilde{M}}$ denotes the canonical bundle on $\widetilde{M}$.

Proposition 3.1 (cf. Theorem 2.2 and Remark [13]). Under the above situation, there exist positive numbers $c_{0}$ and $\nu_{0}$ (depending only on $X, L$ and $G$ ) with the following properties: 
(1) For any $\nu \geq \nu_{0}$,

$$
c_{0} \nu^{n} d \leq \operatorname{dim} H_{L^{2}(U \cap R)}^{0}\left(\widetilde{M}, \mathcal{O}_{\widetilde{M}}\left(\pi^{*} E_{\nu} \otimes K_{\widetilde{M}}\right)\right)
$$

(2) For any $\nu \geq \nu_{0}$,

$$
\begin{aligned}
c_{0} \nu^{n}(d-1) \leq & \operatorname{dim} H_{L^{2}(U \cap R)}^{0}\left(\widetilde{M}, \mathcal{O}_{\widetilde{M}}\left(\pi^{*} E_{\nu} \otimes K_{\widetilde{M}}\right)\right) \\
& -\operatorname{dim} H^{0}\left(X, \mathcal{O}_{X}\left(E_{\nu} \otimes K_{X}\right)\right) .
\end{aligned}
$$

Remark 3.2. In [13, Theorem 2.2], $G$ is assumed to admit a smooth metric with semi-positive curvature, but we do not assume that $G$ admits such metrics.

Proof. Since $L$ is a big line bundle, some positive multiple $L^{m_{0}}$ of $L$ can be written as the tensor product of an ample line bundle $A$ and an effective line bundle $F$. We can take a smooth metric $h_{A}$ on $A$ such that the Chern curvature is a Kähler form on the regular locus $X_{\text {reg }}$ of $X$ since $A$ is ample. Further we take the singular metric $h_{F}$ on $F$ constructed from sections of $F$. Note that the curvature current of $h_{F}$ is (semi-)positive as $(1,1)$-currents on $X_{\text {reg }}$. Let $\omega$ be the Kähler form on $X_{\text {reg }}$ defined by

$$
\omega:=\frac{1}{m_{0}} \sqrt{-1} \Theta_{h_{A}}(A)
$$

Then the curvature current $\sqrt{-1} \Theta_{h}(L)$ of $h$ satisfies $\sqrt{-1} \Theta_{h}(L) \geq \omega>0$ since the curvature current of $h_{F}$ is semi-positive. Here $h$ is the metric on $L$ which is defined by $\left(h_{A} \otimes h_{F}\right)^{1 / m_{0}}$.

Fix a point $p$ in $M$ such that $h$ is smooth on a neighborhood of $p$. Further we take local coordinates $\left(z_{1}, \ldots, z_{n}\right)$ centered at $p$ and a function $\varphi_{p}$ on $X$ with the following properties:

(a) The support of $\varphi_{p}$ is contained in a (small) neighborhood of $p$.

(b) $\varphi_{p}=\log \left(\left|z_{1}\right|^{2}+\cdots+\left|z_{n}\right|^{2}\right)$ on a smaller neighborhood of $p$.

(c) $\varphi_{p}$ is smooth except at $p$.

We take a smooth metric $k$ on $G$. Since the curvature of $h$ is strictly positive, there exists a sufficiently large integer $a_{0}$ with the following properties on 
$X_{\text {reg }}$

$$
\begin{aligned}
& \frac{a_{0}}{2} \omega+\sqrt{-1} \Theta_{k}(G) \geq \omega, \\
& a_{0} \sqrt{-1} \Theta_{h}(L)+d d^{c} \varphi_{p} \geq \frac{a_{0}}{2} \omega .
\end{aligned}
$$

The regular locus $X_{\text {reg }}$ is not compact, but we can take $a_{0}$ with the above properties since the support of $\varphi_{p}$ is compact.

Now we fix local trivializations of $L$ and $G$ on a neighborhood of $p$. Since $\pi$ is an étale covering, the coordinate $\left(z_{1}, \ldots, z_{n}\right)$ determines the local coordinates on the neighborhoods of points in the fiber $\pi^{-1}(p)$ over $p$, which give the local trivialization of $K_{\widetilde{M}}$. Under these trivializations, a $\pi^{*} E_{\nu}$-valued $(n, 0)$-form $s$ can be seen as the holomorphic function on a neighborhood of $\pi^{-1}(p)$. Therefore for a multi-index $\alpha=\left(\alpha_{1}, \ldots, \alpha_{n}\right) \in \mathbb{Z}_{\geq 0}^{n}$, the differential $\left(\partial^{|\alpha|} s / \partial z^{\alpha}\right)$ can be defined on a neighborhood of $\pi^{-1}(p)$, where $|\alpha|$ is the norm of $\alpha$ which is defined by $|\alpha|=\sum_{i=1}^{n} \alpha_{i}$.

Fix an integer $\nu_{0}$ with $\nu_{0}>n a_{0}$. Then we construct holomorphic $\pi^{*} E_{\nu^{-}}$ valued $(n, 0)$-forms for any $\nu \geq \nu_{0}$ by using solutions of the $\bar{\partial}$-equation with $L^{2}$-estimate.

Claim 3.3. For a multi-index $\alpha=\left(\alpha_{1}, \ldots, \alpha_{n}\right)$ with $|\alpha| \leq \nu / a_{0}-n$ and $a$ point $q$ in $\pi^{-1}(p)$, there exists a holomorphic $\pi^{*} E_{\nu}$-valued $(n, 0)$-form $s_{\alpha, q}$ with the following properties:

(1) For any multi-index $\beta$ with $|\beta| \leq \nu / a_{0}-n$ and a point $r$ in $\pi^{-1}(p)$,

$$
\frac{\partial^{|\beta|} s_{\alpha, q}}{\partial z^{\beta}}(r)=\left\{\begin{array}{cc}
1 & \text { if } r=q \text { and } \beta=\alpha \\
0 & \text { others }
\end{array}\right.
$$

$(2)$

$$
\int_{\widetilde{M}}\left|s_{\alpha, q}\right|_{\pi^{*}\left(h^{\nu} \otimes k\right), \pi^{*} \omega}^{2}\left(\pi^{*} \omega\right)^{n}<\infty .
$$

Proof. First we take a smooth $\pi^{*} E_{\nu}$-valued $(n, 0)$-form $u$ with the following properties:

(a) For a multi-index $\beta$ with $|\beta| \leq \nu / a_{0}-n$ and a point $r$ in $\pi^{-1}(p)$,

$$
\frac{\partial^{|\beta|} u}{\partial z^{\beta}}(r)=\left\{\begin{array}{cc}
1 & \text { if } r=q \text { and } \beta=\alpha, \\
0 & \text { others. }
\end{array}\right.
$$


(b) The support of $u$ is contained in a (small) neighborhood of $q$.

(c) $u$ is holomorphic on a smaller neighborhood of $q$.

Then we obtain solutions of the $\bar{\partial}$-equation $\bar{\partial} \eta=\bar{\partial} u$ by applying [5, Théorèm 4.1]. In order to obtain solutions of this equation with $L^{2}$-estimate, we need to compute the curvature of $\pi^{*} E_{\nu}$ and the $L^{2}$-norm of $\bar{\partial} u$ with respect to the suitable metric.

First we see that the curvature of the metric on $\pi^{*} E_{\nu}$ defined by $\pi^{*}\left(h^{\nu} \otimes\right.$ $k) \exp \left(-\frac{\nu}{a_{0}} \pi^{*} \varphi_{p}\right)$ is strictly positive. By the choice of $a_{0}$ and $\nu_{0}$, we obtain

$$
\begin{aligned}
& \sqrt{-1} \Theta_{\pi^{*}\left(h^{\nu} \otimes k\right) \exp \left(-\frac{\nu}{a_{0}} \pi^{*} \varphi_{p}\right)}\left(\pi^{*} E_{\nu}\right) \\
= & \nu \sqrt{-1} \Theta_{\pi^{*} h}\left(\pi^{*} L\right)+\sqrt{-1} \Theta_{\pi^{*} k}\left(\pi^{*} G\right)+\frac{\nu}{a_{0}} d d^{c} \pi^{*} \varphi_{p} \\
\geq & \frac{\nu}{2} \pi^{*} \omega+\sqrt{-1} \Theta_{\pi^{*} k}\left(\pi^{*} G\right) \\
\geq & \pi^{*} \omega
\end{aligned}
$$

for any $\nu \geq \nu_{0}$.

Now we compute the $L^{2}$-norm of $\bar{\partial} u$ with respect to the above metric. The support of $\bar{\partial} u$ does not contain the pole of $\pi^{*} \varphi_{p}$ since $u$ is holomorphic on the neighborhood of $q$. Further $\pi^{*} h$ is smooth at $q$ by the choice of $p$. Therefore we obtain

$$
\int_{\widetilde{M}}|\bar{\partial} u|_{\pi^{*}\left(h^{\nu} \otimes k\right), \pi^{*} \omega}^{2} \exp \left(-\frac{\nu}{a_{0}} \pi^{*} \varphi_{p}\right)\left(\pi^{*} \omega\right)^{n}<\infty .
$$

Since $X$ is projective, $M=X \backslash R$ admits a complete Kähler metric. It implies that the étale covering $\widetilde{M}$ also admits a complete Kähler metric. Hence by applying [5, Théorèm 4.1], we obtain a $\pi^{*} E_{\nu \text {-valued }}(n, 0)$-form $\eta$ such that

$$
\begin{aligned}
& \bar{\partial} \eta=\bar{\partial} u \\
& \int_{\widetilde{M}}|\eta|_{\pi^{*}\left(h^{\nu} \otimes k\right), \pi^{*} \omega}^{2} \exp \left(-\frac{\nu}{a_{0}} \pi^{*} \varphi_{p}\right)\left(\pi^{*} \omega\right)^{n}<\infty .
\end{aligned}
$$

Then the $\pi^{*} E_{\nu}$-valued $(n, 0)$-form $s_{\alpha, q}$ defined by $s_{\alpha, q}:=u-\eta$ is holomorphic and satisfies property (2) in the claim. The $L^{2}$-estimate and the choice of $\varphi_{p}$ imply that $\eta$ vanishes at any point in $\pi^{-1}(p)$ to the order $\left(\nu / a_{0}\right)-n$. Therefore $s_{\alpha, q}$ satisfies property (1) in the claim by the choice of $u$.

We see that $s_{\alpha, q}$ satisfies $L^{2}$-condition $(*)$ in Proposition 3.1. Fix trivializations of $L$ and $G$ on a neighborhood $V_{r}$ of $r \in U \cap R$. Under these 
trivializations, $h$ can be seen as the function bounded below on $V_{r}$, since the curvature of $h$ is positive. Here we implicitly used the known fact that an almost plurisubharmonic is upper semi-continuous. By Claim 3.3, we have

$$
\int_{\pi^{-1}\left(V_{r}\right)}\left|s_{\alpha, q}\right|_{\pi^{*} \omega}\left(\pi^{*} \omega\right)^{n}<\infty
$$

Here we regard $s_{\alpha, q}$ as the holomorphic $(n, 0)$-form on $\pi^{-1}\left(V_{r}\right)$. By simple computations we obtain

$$
\left|s_{\alpha, q}\right|_{\widetilde{\omega}}^{2} \frac{\widetilde{\omega}^{n}}{n !}=\sqrt{-1} n^{2} s_{\alpha, q} \wedge \overline{s_{\alpha, q}}
$$

for any Hermitian form $\widetilde{\omega}$. Therefore $s_{\alpha, q}$ satisfies $L^{2}$-condition $(*)$.

Finally we show estimates (1) and (2) in Proposition 3.1. Let $V_{\nu}$ be the $\mathbb{C}$-vector space which is spanned by $\left\{s_{\alpha, q}\right\}_{\alpha, q}$, where $\alpha$ runs through multiindices with $|\alpha| \leq\left(\nu / a_{0}\right)-n$ and $q$ runs through the points in the fiber $\pi^{-1}(p)$ over $p$. Since $s_{\alpha, q}$ satisfies $L^{2}$-condition $(*)$, we have

$$
V_{\nu} \subset H_{L^{2}(U \cap R)}^{0}\left(\widetilde{M}, \mathcal{O}_{\widetilde{M}}\left(\pi^{*} E_{\nu} \otimes K_{\widetilde{M}}\right)\right)
$$

We can easily show that sections $\left\{s_{\alpha, q}\right\}_{\alpha, q}$ are linearly independent from property (1) in Claim 3.3. Thus, the dimension of $V_{\nu}$ is equal to the number of pairs of multi-index $\alpha$ with $|\alpha| \leq\left(\nu / a_{0}\right)-n$ and $q \in \pi^{-1}(p)$, which implies that the dimension is greater than or equal to $c_{0} \nu^{n} d$ for some number $c_{0}$ depending only on $a_{0}$ and $n$. Therefore we obtain estimate (1) in Proposition 3.1.

It remains to show estimate (2). The pull-back $\pi^{*} t$ of a holomorphic $E_{\nu}$-valued $(n, 0)$-form $t$ on $X$ satisfies $L^{2}$-condition $(*)$ since $t$ is globally defined on $X$. Thus we obtain

$$
\pi^{*} H^{0}\left(X, \mathcal{O}_{X}\left(E_{\nu} \otimes K_{X}\right)\right) \subset H_{L^{2}(U \cap R)}^{0}\left(\widetilde{M}, \mathcal{O}_{\widetilde{M}}\left(\pi^{*} E_{\nu} \otimes K_{\widetilde{M}}\right)\right)
$$

Fix a point $q_{0}$ in the fiber $\pi^{-1}(p)$ over $p$. Let $W_{\nu}$ be the $\mathbb{C}$-vector space which is spanned by $\left\{s_{\alpha, q}\right\}_{\alpha, q \neq q_{0}}$. By property (1) in Claim 3.3, we can show that $\left\{s_{\alpha, q}\right\}_{\alpha, q \neq q_{0}}$ are orthogonal to $\pi^{*} H^{0}\left(X, \mathcal{O}_{X}\left(E_{\nu} \otimes K_{X}\right)\right)$. Further the dimension of $W_{\nu}$ is greater than or equal to $c_{0} \nu^{n}(d-1)$. Therefore we obtain estimate (2) in Proposition 3.1. 


\subsection{On formal schemes}

In the proof of Theorem 3.6, we need to consider formal schemes (formal completions) and cohomology groups on them. In this subsection, we recall basic facts on formal schemes. Further we give a proof of Proposition 3.4, which is applied for the proof of Theorem 3.6.

Let $X$ be an irreducible (not necessarily compact) analytic space and $Y$ be an analytic subspace of $X$ with the ideal sheaf $\mathcal{I}:=\mathcal{I}_{Y}$. Note that $Y$ is not necessarily irreducible and reduced. We denote by $Y_{k}$, the $k$-th infinitesimal neighborhood of $Y$ defined by $\mathcal{I}^{k}$. Namely, $Y_{k}$ is the analytic space with the structure sheaf $\mathcal{O}_{Y_{k}}:=\mathcal{O}_{X} / \mathcal{I}^{k}$. The ringed space defined by

$$
\left(\widehat{X}, \mathcal{O}_{\widehat{X}}\right)=\left(Y, \lim _{\leftarrow} \mathcal{O}_{X} / \mathcal{I}^{k}\right)
$$

is called the formal completion of $X$ with respect to $Y$. For a given coherent sheaf $\mathcal{F}$ on $X$, the associated analytic sheaf $\widehat{\mathcal{F}}$ with respect to $Y$ is defined by

$$
\widehat{\mathcal{F}}:=\lim _{\leftarrow} \mathcal{F} \otimes \mathcal{O}_{X} / \mathcal{I}^{k}
$$

which is an $\mathcal{O}_{\widehat{X}}$-module sheaf. The cohomology groups of $\widehat{\mathcal{F}}$ may be an infinite-dimensional space even if $Y$ is compact, but under a suitable condition on $Y$, these cohomology groups are finite dimensional.

Proposition 3.4. Let $X$ be an irreducible (not necessarily compact) analytic space, $Y$ be a compact analytic subspace of $X$ with the ideal sheaf $\mathcal{I}$, and $E$ be a locally free sheaf on $X$. Assume that there exists a positive integer $k_{0}$ such that

$$
H^{0}\left(X, \mathcal{O}_{X}(E) \otimes \mathcal{I}^{k} / \mathcal{I}^{k+1}\right)=0 \quad \text { for } k \geq k_{0}
$$

Then $H^{0}\left(\widehat{X}, \widehat{\mathcal{O}_{X}(E)}\right)$ injects into $H^{0}\left(Y, \mathcal{O}_{Y_{k_{0}}}(E)\right)$. Further for a sufficiently large $k$ we have

$$
H^{0}\left(\widehat{X}, \widehat{\mathcal{O}_{X}(E)}\right)=H^{0}\left(X, \mathcal{O}_{Y_{k}}(E)\right)
$$

Remark 3.5. When $Y$ is a locally complete intersection, we have the isomorphism $\mathcal{I}^{k} / \mathcal{I}^{k+1} \cong S^{k} N_{Y / X}^{*}$ as an $\mathcal{O}_{X}$-module. Then, if the normal bundle $N_{Y / X}$ satisfies some positivity conditions (such as ampleness), the assumption in Proposition 3.4 is satisfied (see, e.g. [10, Proposition 6.3.14]). 
Proof. For every positive integer $k$, we have the exact sequence

$$
0 \longrightarrow \mathcal{O}_{X}(E) \otimes \mathcal{I}^{k} / \mathcal{I}^{k+1} \longrightarrow \mathcal{O}_{Y_{k+1}}(E) \longrightarrow \mathcal{O}_{Y_{k}}(E) \longrightarrow 0 .
$$

By the induced long exact sequence and the assumption, we know that the map

$$
H^{0}\left(X, \mathcal{O}_{Y_{k+1}}(E)\right) \rightarrow H^{0}\left(X, \mathcal{O}_{Y_{k}}(E)\right)
$$

is injective for $k \geq k_{0}$. It implies that the map $H^{0}\left(X, \mathcal{O}_{Y_{k}}(E)\right) \rightarrow H^{0}(X$, $\left.\mathcal{O}_{Y_{k_{0}}}(E)\right)$ is injective for $k \geq k_{0}$. Therefore we obtain

$$
H^{0}\left(\widehat{X}, \widehat{\mathcal{O}_{X}(E)}\right)=\lim _{k} H^{0}\left(X, \mathcal{O}_{Y_{k}}(E)\right) \subset H^{0}\left(X, \mathcal{O}_{Y_{k_{0}}}(E)\right) .
$$

The first equality above follows from general facts about formal schemes (see, e.g. [8, Proposition 9.2]).

Now we prove the latter conclusion. By the above argument, we have already known that the dimensions of the cohomology groups

$$
\left\{\operatorname{dim} H^{0}\left(X, \mathcal{O}_{Y_{k}}(E)\right)\right\}_{k \geq k_{0}}
$$

are decreasing for $k \geq k_{0}$. The dimensions of the cohomology groups are finite since $Y$ is compact. Therefore the cohomology groups must be isomorphic for a sufficiently large $k$. It implies that

$$
\left.H^{0}\left(\widehat{X}, \widehat{\mathcal{O}_{X}(E}\right)\right)=\lim _{k} H^{0}\left(Y, \mathcal{O}_{Y_{k}}(E)\right)=H^{0}\left(Y, \mathcal{O}_{Y_{k}}(E)\right)
$$

for a sufficiently large $k$.

\subsection{Proof of Theorem 3.6}

In this subsection, we prove the following theorem, which leads to Corollary 1.4 .

Theorem 3.6. Let $Y$ be a connected analytic subset of a smooth projective variety $X$ of dimension $n, U$ be a connected neighborhood of $Y$, and $R$ be an analytic subset of $X$. If the cohomological dimension of the complement $X \backslash Y$ is smaller than or equal to $(n-2)$, then the map

$$
j_{*}: \pi_{1}(U \backslash R) \longrightarrow \pi_{1}(X \backslash R)
$$

is surjective. In particular, if $R=\phi$, the $\operatorname{map} j_{*}: \pi_{1}(Y) \longrightarrow \pi_{1}(X)$ is surjective. 
First we recall the definition of the cohomological dimension.

Definition 3.7. Let $Y$ be an analytic subset of a projective variety $X$. The (algebraic) cohomological dimension of the complement $X \backslash Y$ is the smallest integer $q$ such that $H^{i}(X \backslash Y, \mathcal{F})=0$ for any $i>q$ and any (algebraic) coherent sheaf $\mathcal{F}$ on $X$. We denote by $\operatorname{cd}(X \backslash Y)$ the cohomological dimension of $X \backslash Y$.

Remark 3.8. (1) The same conclusion as Corollary 1.4 holds under the weaker assumption that $\mathcal{O}_{\mathbb{P}\left(E^{*}\right)}(-1)$ is $(n-2)$-ample.

(2) If $X \backslash S$ is $q$-complete, then we can prove that $\pi_{i}(X, S)=0$ for $i \leq n-$ $q-1$ from the same argument as in subsection 2.2. Here a (non-compact) complex manifold is called $q$-complete if the manifold admits a (smooth) exhaustive function whose Levi-form has at least $(n-q)$ positive eigenvalues at any point.

(3) If a complex manifold $Z$ is $q$-complete, then the (analytic) cohomological dimension of $Z$ is smaller than or equal to $q$. It follows from the classical Andreotti-Grauert vanishing theorem (see [1]), but in general the converse fails even if $Z$ is a quasi-projective manifold. Therefore Theorem 3.6 is of value.

Proof of Theorem 3.6. We denote by $M$ the complement $X \backslash R$ and by $d$ the index of the image of $\pi_{1}(U \backslash R)$ in $\pi_{1}(X \backslash R)$ under $j_{*}$. Let $\pi: \widetilde{M} \rightarrow M$ be an étale covering with $\pi_{*}\left(\pi_{1}(\widetilde{M})\right)=j_{*}\left(\pi_{1}(U \backslash R)\right)$. From the construction of the covering, the degree of $\pi$ is equal to $d$. Our goal is to show that $d$ is equal to one. Since $U$ is a connected and smooth open set, the complement $U \backslash R$ is also connected. Hence there exists a holomorphic map $\tilde{j}$ with the following commutative diagram:

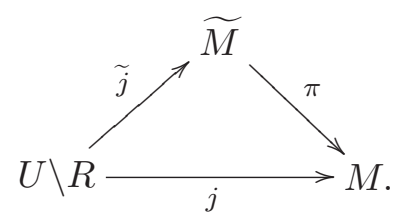

Fix a big (or ample) line bundle $L$ on $X$. Then by applying Proposition 3.1, we obtain positive numbers $c_{0}$ and $\nu_{0}$ such that

$$
\begin{aligned}
c_{0} \nu^{n}(d-1) \leq & \operatorname{dim} H_{L^{2}(U \cap R)}^{0}\left(\widetilde{M}, \mathcal{O}_{\widetilde{M}}\left(\pi^{*} E_{\nu} \otimes K_{\widetilde{M}}\right)\right) \\
& -\operatorname{dim} H^{0}\left(X, \mathcal{O}_{X}\left(E_{\nu} \otimes K_{X}\right)\right)
\end{aligned}
$$

for any $\nu \geq \nu_{0}$. 
Lemma 3.9. In the above situation, we have

$$
\operatorname{dim} H_{L^{2}(U \cap R)}^{0}\left(\widetilde{M}, \mathcal{O}_{\widetilde{M}}\left(\pi^{*} E_{\nu} \otimes K_{\widetilde{M}}\right)\right) \leq \operatorname{dim} H^{0}\left(\widehat{U}, \mathcal{O}_{U}\left(\widehat{E_{\nu} \otimes} K_{U}\right)\right)
$$

Proof. If $s$ is a holomorphic $\pi^{*} E_{\nu}$-valued $(n, 0)$-form on $\widetilde{M}$ with bounded $L^{2}$-norm $\left(L^{2}\right.$-condition $(*)$ in Proposition 3.1$)$. Then the pull-back $\widetilde{j}^{*}(s)$ is

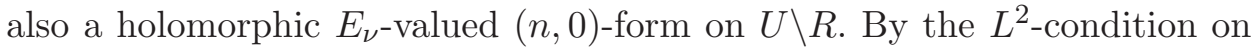
$U \cap R$, the pull-back $\widetilde{j}^{*}(s)$ is $L^{2}$-bounded on neighborhoods of points in $R$, thus $\widetilde{j}^{*}(s)$ can be extended to the holomorphic section on $U$ by the Riemann extension theorem. It implies that

$$
\operatorname{dim} H_{L^{2}(U \cap R)}^{0}\left(\widetilde{M}, \mathcal{O}_{\widetilde{M}}\left(\pi^{*} E_{\nu} \otimes K_{\widetilde{M}}\right)\right) \leq \operatorname{dim} H^{0}\left(U, \mathcal{O}_{U}\left(E_{\nu} \otimes K_{U}\right)\right) .
$$

Note that $\mathcal{O}_{U}\left(E_{\nu} \otimes K_{X}\right)$ is a locally free sheaf since $X$ is smooth. Hence the natural map

$$
H^{0}\left(U, \mathcal{O}_{U}\left(E_{\nu}\right) \otimes K_{U}\right) \rightarrow H^{0}\left(\widehat{U}, \mathcal{O}_{U}\left(\widehat{E_{\nu} \otimes} K_{U}\right)\right)
$$

is injective. It follows from general facts on formal completions. This completes the proof.

Now we have

$$
\begin{aligned}
& H^{0}\left(\widehat{U}, \mathcal{O}_{U}\left(\widehat{E_{\nu} \otimes} K_{U}\right)=\lim _{k} H^{0}\left(U, \mathcal{O}_{Y_{k}}\left(E_{\nu} \otimes K_{U}\right)\right),\right. \\
& H^{0}\left(\widehat{X}, \mathcal{O}_{X}\left(\widehat{E_{\nu} \otimes} K_{X}\right)=\lim _{\leftarrow} H^{0}\left(X, \mathcal{O}_{Y_{k}}\left(E_{\nu} \otimes K_{X}\right)\right)\right.
\end{aligned}
$$

Since the supports of the sheaves on the right-hand sides are contained in $U$, the left-hand sides coincide. By these arguments and Lemma 3.9, we obtain

$$
c_{0} \nu^{n}(d-1) \leq \operatorname{dim} H^{0}\left(\widehat{X}, \mathcal{O}_{X}\left(\widehat{E_{\nu} \otimes} K_{X}\right)\right)-\operatorname{dim} H^{0}\left(X, \mathcal{O}_{X}\left(E_{\nu} \otimes K_{X}\right)\right) .
$$

Our goal is to show that the right-hand side of (3.1) is equal to zero. For this goal, we estimate the right-hand side by using the formal duality theorem.

Claim 3.10. The right-hand side of (3.1) can be estimated from above by the dimension of $H^{n-1}\left(X \backslash Y, \mathcal{O}_{X}\left(E_{\nu}^{*}\right)\right)$.

Before the proof, we see that this claim implies that $d$ is equal to one. By the assumption, the cohomological dimension of $X \backslash Y$ is smaller than or 
equal to $(n-2)$, thus we have

$$
H^{n-1}\left(X \backslash Y, \mathcal{O}_{X}\left(E_{\nu}^{*}\right)\right)=0
$$

Then by inequality (3.1), we obtain $d=1$.

It remains to prove the above claim for the proof of Theorem 3.6.

Proof of Claim 3.10. In the proof, we use the theory of local cohomologies. By considering the long exact sequence of local cohomologies (see [7]), we obtain

$$
\begin{gathered}
\cdots \rightarrow H_{Y}^{n-i}\left(X, \mathcal{O}_{X}\left(E_{\nu}^{*}\right)\right) \rightarrow H^{n-i}\left(X, \mathcal{O}_{X}\left(E_{\nu}^{*}\right)\right) \rightarrow H^{n-i}\left(X \backslash Y, \mathcal{O}_{X}\left(E_{\nu}^{*}\right)\right) \\
\rightarrow H_{Y}^{n-i+1}\left(X, \mathcal{O}_{X}\left(E_{\nu}^{*}\right)\right) \rightarrow H^{n-i+1}\left(X, \mathcal{O}_{X}\left(E_{\nu}^{*}\right)\right) \\
\quad \rightarrow H^{n-i+1}\left(X \backslash Y, \mathcal{O}_{X \backslash Y}\left(E_{\nu}^{*}\right)\right) \rightarrow \cdots
\end{gathered}
$$

We investigate the local cohomology $H_{Y}^{n-i+1}\left(X, \mathcal{O}_{X}\left(E_{\nu}^{*}\right)\right)$. Then we have

$$
\begin{aligned}
H_{Y}^{n-i+1}\left(X, \mathcal{O}_{X}\left(E_{\nu}^{*}\right)\right) & =\underset{\vec{k}}{\lim } \operatorname{Ext}^{n-i+1}\left(\mathcal{O}_{Y_{k}}, \mathcal{O}_{X}\left(E_{\nu}^{*}\right)\right) \\
& =\underset{\vec{k}}{\lim } \operatorname{Ext}^{n-i+1}\left(\mathcal{O}_{Y_{k}}\left(E_{\nu} \otimes K_{X}\right), \mathcal{O}_{X}\left(K_{X}\right)\right)
\end{aligned}
$$

The Serre duality theorem on $X$ implies that

$$
\operatorname{Ext}^{n-i+1}\left(\mathcal{O}_{Y_{k}}\left(E_{\nu} \otimes K_{X}\right), \mathcal{O}_{X}\left(K_{X}\right)\right)
$$

is equal to the dual space of

$$
H^{i-1}\left(X, \mathcal{O}_{Y_{k}}\left(E_{\nu} \otimes K_{X}\right)\right)
$$

The inverse limit of the above cohomology groups is equal to the cohomology of the formal completion with respect to $Y$. Therefore we obtain

$$
H_{Y}^{n-i+1}\left(X, \mathcal{O}_{X}\left(E_{\nu}^{*}\right)\right)=H^{i-1}\left(\widehat{X}, \mathcal{O}_{X}\left(\widehat{E_{\nu} \otimes} K_{X}\right)\right)^{*}
$$

On the other hand, by using the Serre duality theorem on $X$ again, we obtain

$$
H^{n-i+1}\left(X, \mathcal{O}_{X}\left(E_{\nu}^{*}\right)\right)=H^{i-1}\left(X, \mathcal{O}_{X}\left(E_{\nu} \otimes K_{X}\right)\right)^{*}
$$

Hence, the long exact sequence above agrees with

$$
\begin{gathered}
\rightarrow H^{n-i}\left(X \backslash Y, \mathcal{O}_{X \backslash Y}\left(E_{\nu}^{*}\right)\right) \rightarrow H^{i-1}\left(\widehat{X}, \mathcal{O}_{X}\left(\widehat{E_{\nu} \otimes} K_{X}\right)\right)^{*} \\
\rightarrow H^{i-1}\left(X, \mathcal{O}_{X}\left(E_{\nu} \otimes K_{X}\right)\right)^{*} \rightarrow .
\end{gathered}
$$


The conclusion follows from this exact sequence.

\subsection{Proof of Corollary 1.4}

In this subsection, we give a proof of Corollary 1.4.

Proof of Corollary 1.4. The proof is same as [18]. By Theorem 1.1, we know that $S$ is connected since $E$ is ample and $r \leq n$. It is sufficient to prove $\operatorname{cd}(X \backslash S) \leq n-2$. A section $s \in H^{0}\left(X, \mathcal{O}_{X}(E)\right)$ induces the morphism $s^{*}: \mathcal{O}_{X}\left(E^{*}\right) \rightarrow \mathcal{O}_{X}$. This morphism is a surjective map onto $\mathcal{I}_{S} \subset \mathcal{O}_{X}$ since the codimension of $S$ is equal to the expected codimension $r$. By taking symmetric powers and Proj, we have the embedding

$$
i: \operatorname{Proj}\left(\bigoplus_{k=0}^{\infty} \mathcal{I}_{S}^{k}\right) \hookrightarrow \operatorname{Proj}\left(\bigoplus_{k=0}^{\infty} S^{k} E^{*}\right)
$$

The left-hand side is equal to the blow-up $\mathrm{Bl}_{S}(X)$ of $X$ along $S$ and the right-hand side is equal to $\mathbb{P}\left(E^{*}\right)$. Then we can easily see that $\mathcal{O}_{\mathrm{Bl}_{S}(X)}(F)=$ $i^{*} \mathcal{O}_{\mathbb{P}\left(E^{*}\right)}(-1)$, where $F$ is the exceptional divisor of $\mathrm{Bl}_{S}(X) \rightarrow X$.

Since $E$ is ample, $\mathcal{O}_{\mathbb{P}\left(E^{*}\right)}(-1)$ is $(r-1)$-ample (see [6, Example 2.7]). It follows this line bundle is $(n-2)$-ample by the assumption of $r<n$. Hence the restriction $\mathcal{O}_{\mathrm{Bl}_{S}(X)}(F)$ is also $(n-2)$-ample. Note the restriction to subvarieties of $q$-ample line bundles is also $q$-ample. In general, the cohomological dimension of the complement of the zero locus of sections of $q$-ample line bundles is smaller than or equal to $q$. Therefore we have $\operatorname{cd}\left(\mathrm{Bl}_{S}(X) \backslash F\right) \leq(n-2)$. Since $\mathrm{Bl}_{S}(X) \backslash F$ is isomorphic to $X \backslash S$, we obtain $\operatorname{cd}(X \backslash S) \leq n-2$. Then the conclusion of Corollary 1.4 follows from Theorem 3.6.

\section{Acknowledgments}

The author wishes to express his gratitude to Professor John Christian Ottem for fruitful discussions. He is indebted to Professor Shin Hayashi, Professor Tomohiko Ishida and Professor Tetsuya Ito for teaching him important ideas from topology. He is grateful to Professor Yoshinori Gongyo and Professor Shinnosuke Okawa for discussions on Landman's example. He is supported by the Grant-in-Aid for Young Scientists (B) number 25800051 from JSPS. 


\section{References}

[1] A. Andreotti and H. Grauert, Théorème de finitude pour la cohomologie des espaces complexes, Bull. Soc. Math. France 90 (1962), 193-259.

[2] R. Bott, On a theorem of Lefschetz, Michigan Math. J. 6 (1959), 211-216.

[3] R. Bott, The stable homotopy of the classical groups, Ann. of Math. (2) 70 (1959), 313-337.

[4] F. Campana and H. Flenner, A characterization of ample vector bundles on a curve, Math. Ann. 287(4) (1990), 571-575.

[5] J.-P. Demailly, Estimations $L^{2}$ pour l'opérateur $\bar{\partial}$ d'un fibré vectoriel holomorphe semi-positif au-dessus d'une variété kählérienne complète, Ann. Sci. École Norm. Sup. (4) 15(3) (1982), 457-511.

[6] J.-P. Demailly, T. Peternell and M. Schneider, Holomorphic line bundles with partially vanishing cohomology, Proceedings of the Hirzebruch 65 Conference on Algebraic Geometry (Ramat Gan, 1993), Israel Math. Conf. Proc., 9, Bar-Ilan University, Ramat Gan, 1996, 165-198.

[7] R. Hartshorne, Cohomological dimension of algebraic varieties, Ann. of Math. (2) 88 (1968), 403-450.

[8] R. Hartshorne, Algebraic geometry. Graduate Texts in Mathematics, 52, Springer, New York, 1977.

[9] R. Lazarsfeld, Some applications of the theory of positive vector bundles. Complete intersections (Acireale, 1983), Lecture Notes in Math., 1092, Springer, Berlin, 1984, 29-61.

[10] R. Lazarsfeld, Positivity in algebraic geometry I, II. Ergebnisse der Mathematik und ihrer Grenzgebiete, 48-49, Springer, Berlin, 2004.

[11] S. Matsumura, On the curvature of holomorphic line bundles with partially vanishing cohomology, RIMS Kôkyûroku 1783 (2012), 155-168.

[12] S. Matsumura, Asymptotic cohomology vanishing and a converse to the Andreotti-Grauert theorem on a surface, Ann. Inst. Fourier 63 (2013), at press.

[13] T. Napier and M. Ramachandran, The $L^{2} \bar{\partial}$-method, weak Lefschetz theorems, and the topology of Kähler manifolds, J. Amer. Math. Soc. 11(2) (1998), 375-396. 
[14] C. Okonek, Barth-Lefschetz theorems for singular spaces, J. Reine Angew. Math. 374 (1987), 24-38.

[15] J.-C. Ottem, Ample subvarieties and q-ample divisors, Adv. Math. 229(5) (2012), 2868-2887.

[16] A.-J. Sommese, Concavity theorems, Math. Ann. 235(1) (1978), 37-53.

[17] A.-J. Sommese, Submanifolds of Abelian varieties, Math. Ann. 233(3) (1978), 229-256.

[18] H. Whitney, On singularities of mappings of Euclidean spaces. I. Mappings of the plane into the plane, Ann. of Math. (2) 62 (1955), 374-410.

Department of Mathematics and Computer Sciences

KAGOSHIMA UNIVERSITY

1-21-35 KoORIMOTO

KAGOSHIMA 890-0065

JAPAN

E-mail address: shinichi@sci.kagoshima-u.ac.jp; mshinichi0@gmail.com

ReCEIVED May 14, 2013 